\title{
Influencia del estilo de aprendizaje del docente en el aula
}

\author{
Vidal Carreras, P. ${ }^{\text {, }}$ Canós-Darós, L. , Santandreu-Mascarell, C. ${ }^{\text {, }}$ Guijarro, E. ${ }^{\text {d }}$ \\ ${ }^{a}$ Universitat Politècnica de València, Valencia, Spain, pivicar@omp.upv.es, ${ }^{\mathrm{b}}$ Universitat Politècnica \\ de València, Valencia, Spain, loucada@omp.upv.es, ${ }^{\mathrm{c}}$ Universitat Politècnica de València, Valencia, \\ Spain, crisanma@omp.upv.es, d Universitat Politècnica de València, Valencia, Spain, \\ esguitar@doe.upv.es.
}

\begin{abstract}
Resumen
El concepto de estilos de aprendizaje (estilos cognitivos) aparece en la literatura en los años 50 del siglo XIX en el ámbito de la psicología cognitiva, siendo considerados como la expresión de las formas particulares de los individuos de percepción e información. Desde entonces, muchos autores han propuesto algunas definiciones, enfoques y métodos para analizar estos estilos.
\end{abstract}

Entre los modelos cognitivos encontramos el modelo de aprendizaje de Kolb a través de las experiencias, que relaciona los estilos de aprendizaje con sus procesos. Según este autor, el aprendizaje se define como el proceso de creación de conocimiento a través de la transformación de la experiencia. El modelo sigue un proceso, basado en el ciclo experiencial de Lewin, en el cual una experiencia concreta o situación diaria activa el conocimiento de tal manera que la persona comienza a recolectar, almacenar, procesar y analizar información. Luego, el individuo generaliza los conceptos e internaliza y madura los conocimientos y habilidades adquiridos. Finalmente, el aprendizaje se contrasta para demostrar que puede ser útil en situaciones o experiencias nuevas.

Kolb define cuatro estilos dominantes de estilos de aprendizaje basados en la forma en que se percibe la información (desde la experiencia directa hasta conceptos abstractos) y se procesa (desde el punto de vista práctico al teórico): divergente, convergente, asimilador o analítico y acomodador.

El objetivo de este trabajo es reflexionar sobre la relación que existe entre el estilo de aprendizaje predominante en un docente y la forma en que se diseña la guía docente de las asignaturas que imparte, en particular en lo relativo a la aplicación de distintas metodologías de enseñanza-aprendizaje y/o a los sistemas de evaluación utilizados. 
Palabras clave: Estilos de aprendizaje, Kolb, Docentes, Sistemas de evaluación

\section{Introducción}

El aprendizaje es un proceso de adquisición, es decir, debemos seguir unas determinadas fases para lograr el resultado final. Lo adquirido puede ser un conocimiento nuevo o un conocimiento mejorado de algo ya conocido, habilidades, habilidades y actitudes. En el ámbito educativo el profesor utiliza diferentes metodologías que le permiten al estudiante mejorar y adquirir este conocimiento, habilidades, habilidades y actitudes (Canós-Darós et al., 2018). En este contexto, Alonso et al. (1999) concluyen que los estudiantes aprenden de manera más efectiva cuando se les enseña en base a sus estilos de aprendizaje predominantes, tanto los de los estudiantes como los de los profesores.

Existen diferentes modelos para poder diferenciar entre distintos estilos de aprendizaje, por ejemplo los modelos de Kagan (1980), de Felder-Silverman (1988) o de Gardner (2011), entre otros. Uno de los más populares es el modelo de Kolb, en el que el aprendizaje se define como el proceso de creación de conocimiento a través de la transformación de la experiencia, es decir, el conocimiento se dinamiza a partir de una experiencia vivida (Canós y Mauri, 2005). Luego, la persona recopila, almacena, trata y analiza información de la experiencia (contexto e internalización), adquiere conocimientos y habilidades más complejas y contrasta su aprendizaje con nuevas experiencias (Beck, 2008).

En este modelo, Kolb define cuatro estilos dominantes de estilos de aprendizaje: divergente, convergente, asimilativo o analítico y adaptado (ver Figura 1). Estos estilos se basan en la forma en que se percibe la información desde la experiencia directa hasta conceptos abstractos, y en la forma en que se procesa la información desde la práctica hasta la teoría (Kolb, 1984).

Las personas divergentes son reflexivas, observadoras y les gusta trabajar en grupos para capturar y recopilar información. Por otra parte, para los convergentes lo principal es la aplicación práctica de ideas, la resolución de problemas o la toma de decisiones, para lo cual las relaciones personales o los aspectos sociales no se toman en cuenta. Los asimiladores son personas analíticas, que pueden asimilar una gran cantidad de información y ordenarla de una manera concisa y lógica para desarrollar modelos teóricos. Los acomodadores siempre están dispuestos a experimentar cosas nuevas y llevar a cabo proyectos arriesgados; este tipo de personas tienen en cuenta el aspecto social y el trabajo en equipo. 
Con estas ideas presentes, nos preguntamos si existe alguna relación entre el estilo de aprendizaje predominante en un docente y las metodologías de enseñanza-aprendizaje y/o a los sistemas de evaluación utilizados que utiliza en clase y que están descritos en la guía docente de las asignaturas que imparte.

Así, la estructura de este trabajo queda del siguiente modo. En el apartado 2 se presenta la descripción de la metodología empleada, en el apartado 3 se presentan los resultados y el análisis de los mismos y en el último apartado las conclusiones y líneas de futuro.

\section{Metodología}

Para realizar el trabajo se planteo la realización de una encuesta a una muestra de profesores del departamento de Organización de Empresas de la Universitat Politècnica de València (UPV) localizada en la ciudad de Valencia (España). Las encuestas se realizaron on line mediante la herramienta institucional de la UPV denominada Poliforma-T.

La encuesta se estructura en tres partes claramente diferenciadas. En la primera parte se identifican variables personales del usuario como género, edad, experiencia profesional, etc. La segunda parte era el propio cuestionario de estilo de aprendizaje de Kolb. El cuestionario consta de 12 items para los que el profesor debe ordenar cuatro afirmaciones relacionadas con distintas habilidades de aprendizaje. Una respuesta con un valor 1 representa la manera menos probable de aprender para el que responde; un valor 4 significa que la afirmación describe mejor cómo aprende, pasando por los valores intermedios 2 y 3 . La tercera parte pregunta a los usuarios acerca de su predilección sobre una serie de metodologías de enseñanza-aprendizaje a emplear en el aula. El profesor debía valorararlas de 1 a 5 en función de su predilección, siendo 1 la menos preferida y 5 la más preferida. A continuación se describen las metodologías consideradas en nuestro análisis:

- Lección Magistral (LM): el profesor explica el tema mediante métodos expositivos y verbales.

- Lección Magistral Participativa (LMP): con interacción y participación activa alumnado).

- Resolución de ejercicios y problemas (EjyProb): el alumnado desarrolla soluciones mediante el ejercicio de rutinas, la aplicación de fórmulas o algoritmos, la aplicación de procedimientos de transformación de la información disponible y la interpretación de los resultados.

- Aprendizaje basado en problemas (ABProb): los alumnos, partiendo de un problema diseñado por el profesor, deben resolverlo buscando la información necesaria para comprenderlo y obtener soluciones. 
- Aprendizaje basado en casos (ABCaso): los alumnos, partiendo de una fundamentación teórica del profesor, deben analizan situaciones reales o simuladas planteadas por el profesor.

- Aprendizaje orientado a proyectos (AOProy): se plantea como el aprendizaje basado en problemas pero con la diferencia que la temática la elige el alumno, suele ser más interdisciplinar y no solo enfocado a aprender sino también a hacer.

- Juegos de rol (Rol): simulación en la que los alumnos adaptan roles definidos por el profesor.

- Trabajo en Grupo alumnos (Grupo): cada miembro del grupo es responsable de su trabajo a la vez que actúa cooperativamente con el resto de miembros.

- Presentación Oral alumno/s (PrOral): el alumno expone de forma verbal un trabajo.

- Evaluación Formativa (EvalF): consiste en utilizar el examen como medio de formación explicando al alumnado el porqué de los fallos cometidos.

La muestra estaba formada por 15 profesores, 9 de género masculino y 5 de género femenino. La edad media de la muestra es de 43,1 años y 15,06 años dedicados en la docencia. Los profesores imparten en titulaciones diversas en las que el departamento de Organización de Empresas de la UPV tiene presencia. Las titulaciones son: Grado en Ingenieria de Organización Industrial, Grado en Tecnologías Industriales, Master en Ingeniería Industrial, Grado en Ingenieria Electrónica, Grado en Administración y Dirección de Empresas, Grado en Gestión y Administración Pública, Grado en Turismo.

\section{Resultados y análisis}

En este apartado se analizan los resultados obtenidos. Cabe señalar en primer lugar tal y como muestra la tabla 1 , que de los 15 profesores que participaron en el análisis la mayoria de ellos, 9 profesores, resultaron ser convergentes en su estilo de aprendizaje. Del resto, 5 profesores presentaron un estilo preferentemente asimilador y uno solo, el estilo divergente. La edad media no resulta una variable a destacar, aunque si se aprecia como para la muestra la media en años impartiendo docencia es mayor en los profesores con estilo asimilador.

Tabla 1. Análisis general en función de los estilos de aprendizaje

\begin{tabular}{|lcccc|}
\hline \multicolumn{1}{|c}{ Estilos } & NoProf & $\%$ & Media Edad & Media Años Docencia \\
\hline Asimilador & 5 & $33,3 \%$ & 43,2 & 18,2 \\
Convergente & 9 & $60,0 \%$ & 42 & 14,22 \\
Acomodador & 0 & $0,0 \%$ & - & - \\
Divergente & 1 & $6,7 \%$ & 49 & 7 \\
\hline
\end{tabular}


En este punto se plantean dos partes para presentar los resultados. En una primera etapa se analiza las relaciones que existen entre el estilo de aprendizaje de los profesores y una serie de factores personales. En la segunda etapa se analizan las relaciones entre los estilos de aprendizaje de los profesores y las metodologías de enseñanza aprendizaje empleadas en el aula.

\subsection{Relación estilo aprendizaje y factores personales}

Los factores a analizar son género, experiencia profesional y primera titulación, tal y como se muestra en la figura 1.

Respecto al género de acuerdo con la figura no se aprecia ninguna influencia remarcable, salvo que el único con estilo divergente de la muestra tiene género masculino.

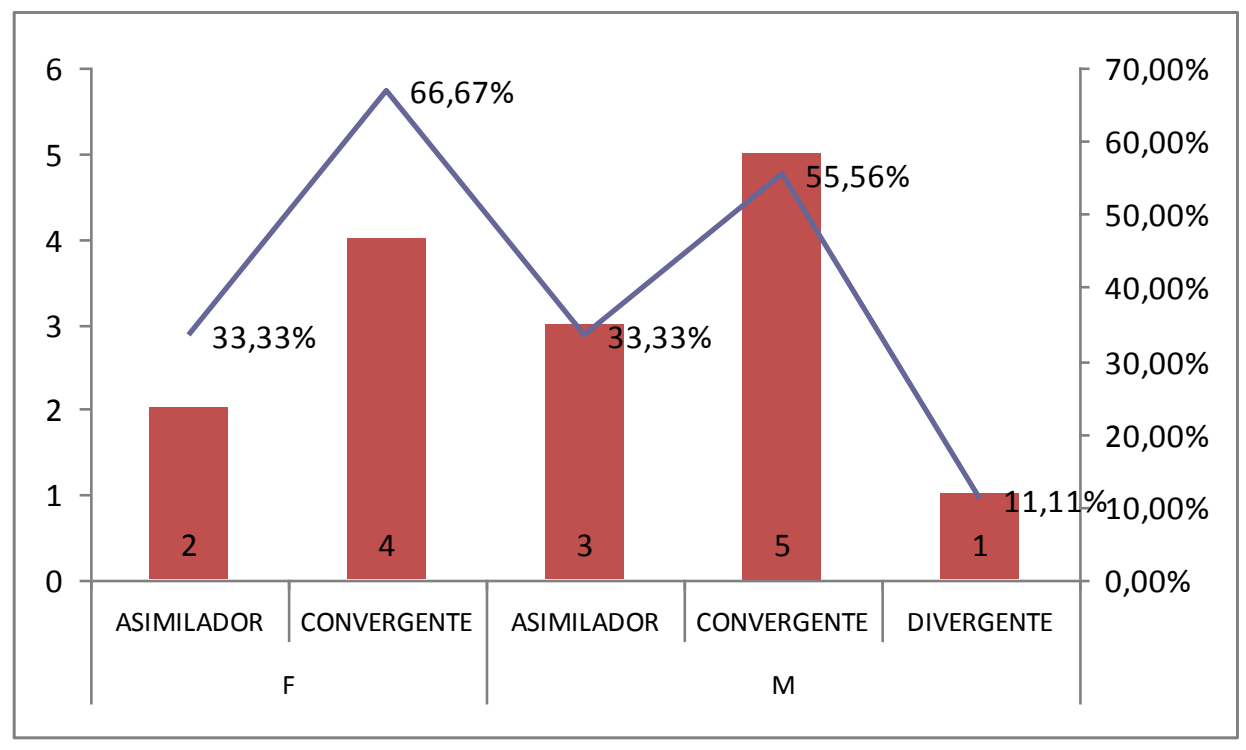

Fig. 1 Relación entre el género y los estilos de aprendizaje

Respecto a la experiencia profesional en ámbitos no relacionados con docencia tampoco se aprecia una influencia considerable. La figura siguiente indica para cada uno de los estilos la distribución entre si según se disponga de experiencia laboral diferente a la docente o no. 


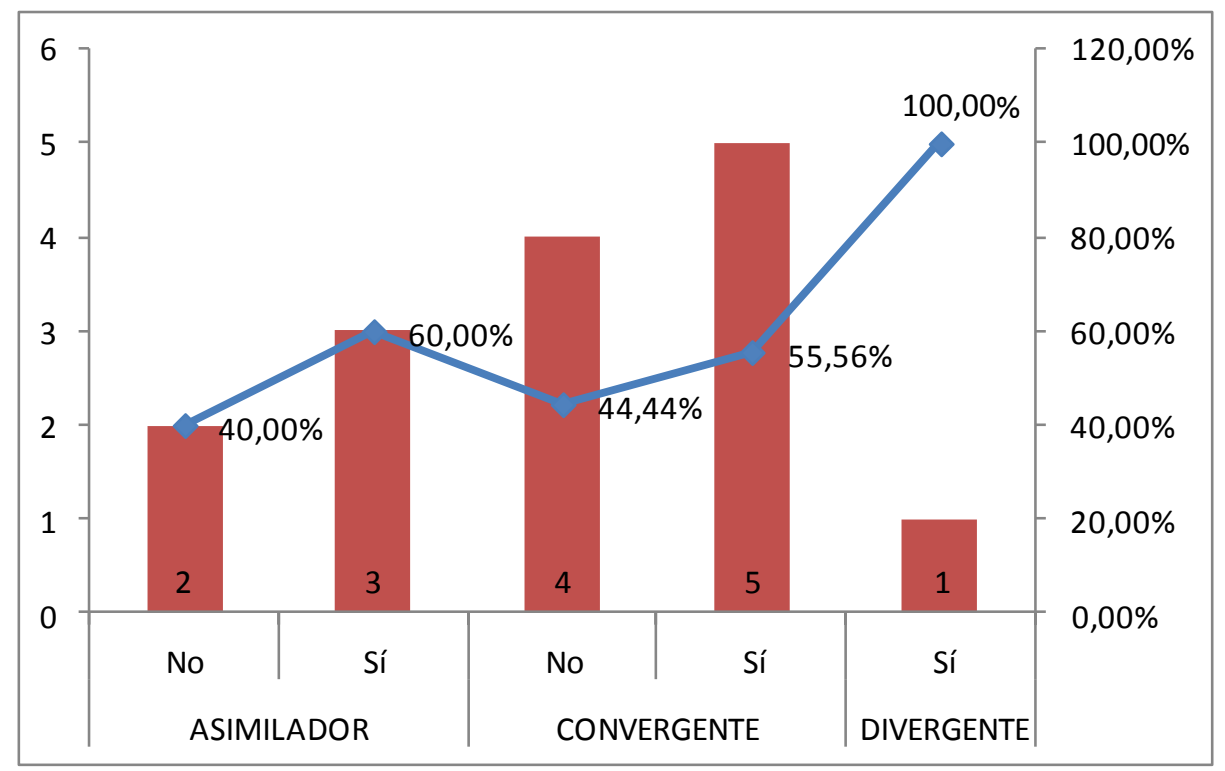

Fig. 2 Relación entre los estilos de aprendizaje y la experiencia profesional

Respecto a la posible influencia de la titulación en el estilo de aprendizaje se observan las siguientes relaciones representadas gráficamente en la figura 3. Se puede señalar una sútil influencia respecto al estilo convergente para los titulados en ADE e Ingeniería Industrial.

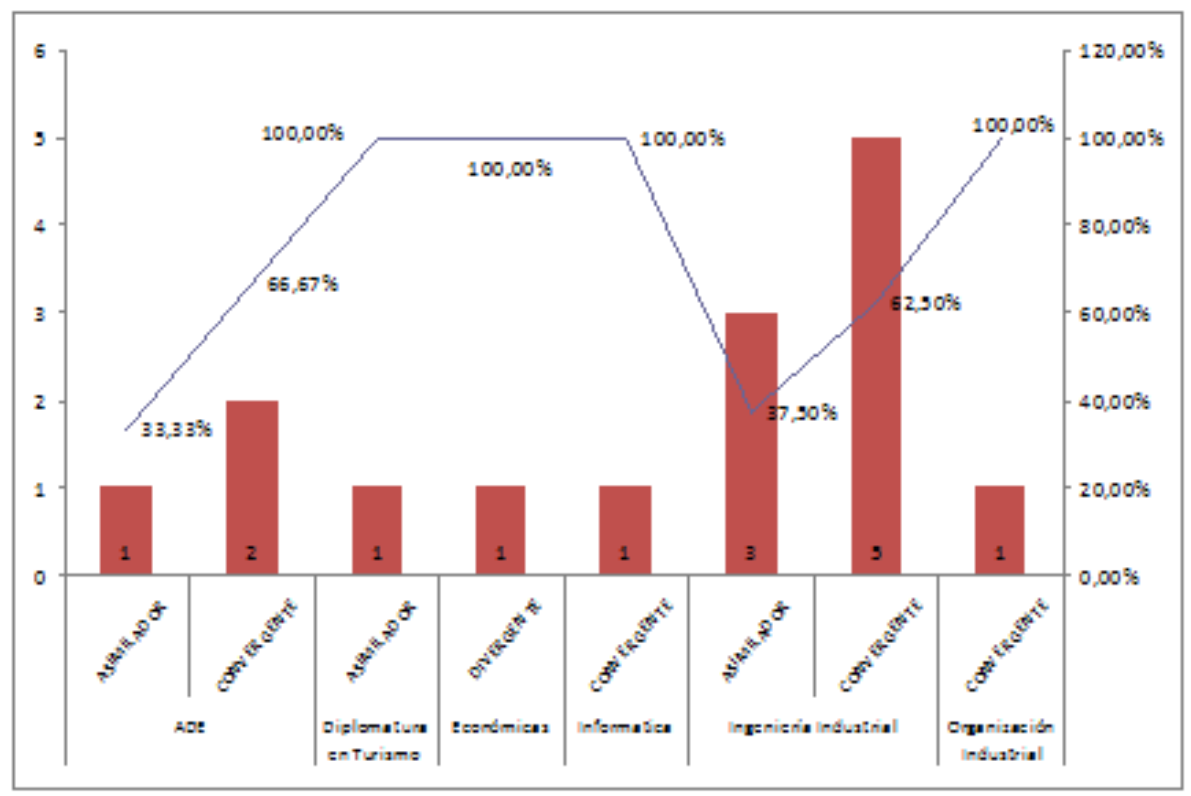

Fig. 3 Relación entre la titulación y los estilos de aprendizaje 


\subsection{Relación estilo aprendizaje y metodologías docentes}

En este apartado se analizan las relaciones entre los estilos de aprendizaje de los profesores y las metodologías de enseñanza aprendizaje empleadas en el aula. Por una parte tenemos los valores agregados de todos los profesores independientemente del estilo de aprendizaje, tal y como se ve en la figura 4.

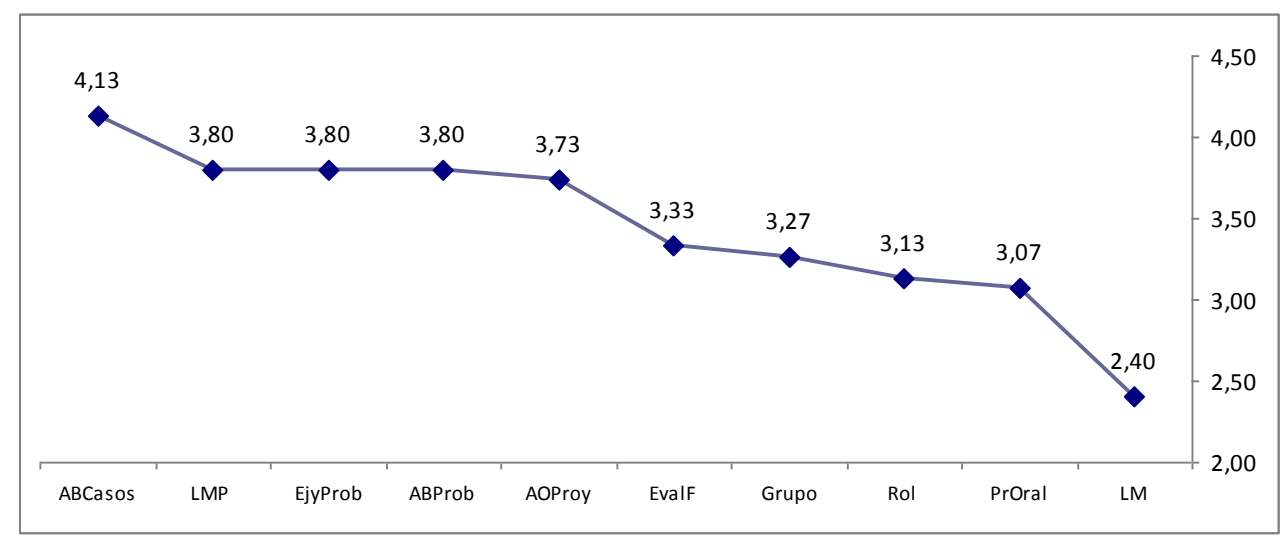

Fig. 4 Valores medios de predilección de metodologías de aprendizaje en el aula

Se puede destacar que el métodos de lección magistral es el menos preferido por los 15 profesores entrevistados, sin embargo el metodo de lección magistral participativa es el segundo más preferido, junto con la realización de problemas y casos, y el aprendizaje basado en problemas. La metodología más preferida es el apredizaje basado en casos.

Por otra parte, en la figura 5 se plantea para cada uno de los estilos de aprendizaje cual es el valor medio de predilección desde 1 (menos preferido) hasta 5 (más preferido) de las metodologías de enseñanza aprendizaje consideradas. 


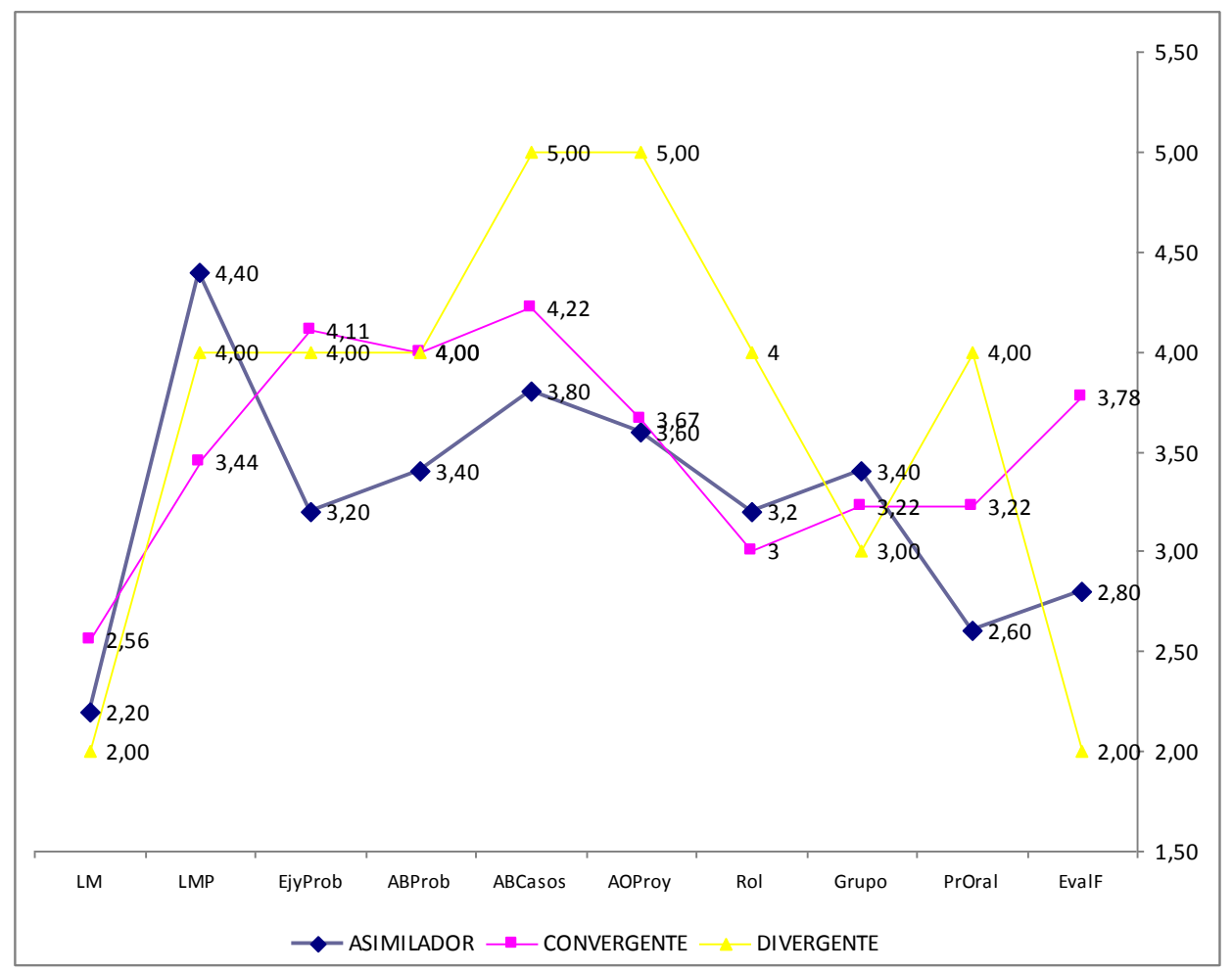

Fig. 5 Relación entre los estilos de aprendizaje y las metodologías de enseñanza-aprendizaje

La metodología preferida por los profesores con un estilo asimilador es la lección magistral participativa seguida por el aprendizaje basado en casos. Las metodologías menos preferidas son la presentación oral y la evaluación formativa. La metodología preferida por los profesores con un estilo convergente es el apredizaje basado en casos, seguida por la realización de problemas y ejercicios y la evaluación formativa. Para el único profesor con enfoque de estilo divergente las mas preferidas son el apredizaje basado en casos y el aprendizaje orientado por proyectos, y las menos preferidas son la lección magistral y la evaluación formativa. Entonces, claramente se observa que si se aprecían influencias en relación al modo de aprender de los profesores de acuerdo con el modelo propuesto por Kolb sobre los estilos de aprendizaje y sus metodologías de enseñanza-aprendizaje preferidas aplicadas en el aula. 


\section{Conclusiones y líneas de futuro}

Este trabajo presenta para una muestra de profesores la influencia que pueden tener determinadas variables en el estilo de aprendizaje del profesor y, por otra parte, la influencia del estilo de aprendizaje del profesor a la hora de aplicar metodologías docentes para impartir la clase.

Respecto a la primera parte se analizó la influencia del género, la experiencia profesional distinta a la universitaria, la titulación y el número de años impartiendo docencia. No se apreciaron influencias significativas de las variables salvo que de modo global es muy destacable que no aparece el estilo de aprendizaje de acomodador en ninguno de los profesores que realizaron la encuesta, y el estilo divergente sólo aparece una vez (6,7\%). El resto de los profesores se reparten entre un un estilo convergente $(60 \%)$ y asimilador $(33,3 \%)$.

Respecto al análisis de la influencia del estilo de aprendizaje del profesor a la hora de impartir la clase en el aula y aplicar ciertas metodologías docentes, se aprecian resultados muy interesantes, puesto que en función del estilo de aprendizaje hay cambios destacables respectos a los modos preferidos de dar la clase en aula.

Como líneas de futuro se plantea ampliar la muestra del profesorado, así como integrar además de los enfoques para la enseñanza en el aula, los enfoques para la evaluación. También sería interesante ver los resultados de profesores de distintos departamentos o analizar los datos de los profesores agrupados por titulaciones para comprobar si hay alguna correspondencia significativa entre estos grupos.

\section{Agradecimientos}

Sistemas de aprendizaje en acción en el ámbito de la educación superior (PIME/2017/A07), Universitat Politècnica de València.

\section{Referencias}

Alonso, C., Gallego, D., \& Honey, P. (1999). Los estilos de aprendizaje. Procedimientos de diagnóstico y mejora. Bilbao: Ediciones Mensajero.

Beck, S. (2008). The teacher's role and approaches in a knowledge society. Cambridge Journal of Education, 38 (4), 465-481.

Canós-Darós, L., \& Mauri-Castelló, J.J. (2005). Metodologías activas para la docencia y aplicación de las nuevas tecnologías: una experiencia. XX Simposium Nacional URSI, Gandía, 2005. 
Canós-Darós, L., Vidal-Carreras, P.I., Garcia-Sabater, J.P., \& Santandreu-Mascarell, C. (2018). Is there a relationship between the lecturer's learning style and methodologies used in teaching? Some reflections. 12th International Technology, Education and Development Conference (INTED 2018)

Felder, R.M., \& Silverman, L.K. (1988). Learning and Teaching Styles. Engineering Education. Engr. Education, 78 (7), 674-681.

Gardner, H. (2011). Frames of mind: The theory of multiple intelligences. Basic books.

Kagan, D.M. (1980). Syntactic complexity and cognitive style. Applied Psycholinguistics, 1 (1), 111122.

Kolb, D.A. (1984). Experiential Learning: experience as the source of learning and development. Englewood Cliffs: Prentice-Hall. 